This article is licensed under the Creative Commons Attribution-NonCommercial 4.0 International License (CC BY-NC) (http://www.karger.com/Services/OpenAccessLicense). Usage and distribution for commercial purposes requires written permission.

\title{
Development and Improvement of Simple Colonic Mucosal Ulcer during Treatment of Severe Ulcerative Colitis with Tacrolimus
}

\author{
Ayumi Ito Bunei Iizuka Teppei Omori Shinichi Nakamura \\ Katsutoshi Tokushige \\ Department of Gastroenterology, Tokyo Women's Medical University, Tokyo, Japan
}

\section{Keywords}

Simple ulcer · Behçet's disease · Ulcerative colitis · Tacrolimus

\begin{abstract}
Diarrhea, melena, and lower abdominal pain developed in a male in his 20 s and colonoscopy showed pancolitis-type severe ulcerative colitis (UC). Treatment was initiated with 4,000 mg of 5 -aminosalicylic acid and $60 \mathrm{mg} /$ day of prednisolone, but the symptoms and inflammatory reaction worsened with prednisolone dose reduction. Tacrolimus was added to the treatment, which subsequently induced remission. Serial colonoscopies during the treatment showed improvement in ulcer and mucosal edema throughout the entire large intestine, but a new solitary round ulcer appeared at the end of the ileum. Since no signs of Behçet's disease were noted, it was considered as a simple ulcer, a complication of UC. Tacrolimus treatment was continued based on continued improvement in clinical features and colonic mucosa, excluding the end of the ileum. Colonoscopy at 6 months after initiation of tacrolimus showed healing of the large intestinal mucosa, although mild congestion was still noted. The
\end{abstract}




\section{Case Reports in Gastroenterology}

Case Rep Gastroenterol 2017;11:168-177

(c) 2017 The Author(s). Published by S. Karger AG, Basel www.karger.com/crg

Ito et al.: Development and Improvement of Simple Colonic Mucosal Ulcer during

Treatment of Severe Ulcerative Colitis with Tacrolimus

solitary round ulcer at the end of the ileum improved to a small erosion. We report the improvement of a simple ulcer that developed during tacrolimus treatment.

(C) 2017 The Author(s)

Published by S. Karger AG, Basel

\section{Introduction}

The ileocecal area is a good site of many inflammatory bowel diseases, from specific enteritis, whose cause can be specified, to nonspecific enteritis, whose cause is unknown, and it is often associated with ulcer. When an ulcer is found in the ileocecal area, it is necessary to distinguish between infectious enterocolitis and inflammatory bowel disease. Therefore, detailed interview, examination, fecal culture, gastrointestinal endoscopy, and whole body examination are necessary.

Differentiation from Behçet's disease (BD) is important in a lesion that creates an ulcer in the ileocecal area. BD presents various systemic symptoms such as oral cavity aftertaste, nodular erythema, or iridocyclitis and is sometimes difficult to treat. There is intestinal tract $\mathrm{BD}$ as a partial disease of $\mathrm{BD}$, and deeply digging ulcerative lesions are typically found in the ileocecal area. In the intestinal tract BD, findings such as oral cavity aftertaste, nodular erythema, and iridocyclitis as described in the diagnostic criteria are confirmed. On the other hand, simple ulcer does not satisfy the diagnostic criteria of BD; it is a case in which ulcerative lesion appears in the ileocecal area, and the presence or absence of diagnostic criteria is important for distinguishing between simple ulcer and intestinal tract BD [1].

On the other hand, ulcerative colitis (UC) is a chronic inflammatory bowel disease exhibiting gastrointestinal symptoms such as diarrhea, bronchial abdominal pain, and abdominal pain. In lesions, ulcers are seen in continuity and diffuse from the rectum, and characteristic histological findings are observed [2]. Many are mild to moderate UC, but there are also steroid resistance and dependent refractory UC, which can be difficult to treat.

We encountered a patient in whom simple ulcer developed and improved during the initial treatment of UC with tacrolimus.

\section{Case Presentation}

The patient was a male in his 20s. His chief complaint was diarrhea, melena, and lower abdominal pain. He had no particular family medical and lifestyle histories.

The patient had been previously healthy. Diarrhea 5-6 times a day had appeared 1 month earlier. Two weeks earlier, the patient had decided to visit the family physician following increase in the frequency of diarrhea to 8-10 times a day, accompanied by melena 5 times/day. UC was suspected, and the patient was referred to our hospital for further management. Colonoscopy was performed at our hospital. Since mucosal edema was severe throughout the large intestine, the colonoscopist focused on the transverse colon. Extensive ulceration or undermining ulcer, reduction of visible vascular patterns, and hemorrhagic tendency were noted in the transverse colon compared to the rectum (Fig. 1). Based on the 


\section{Case Reports in Gastroenterology}

Case Rep Gastroenterol 2017;11:168-177

(c) 2017 The Author(s). Published by S. Karger AG, Basel www.karger.com/crg

to al: Development and Improvement of Simple Colonic Mucosal Ulcer during

Treatment of Severe Ulcerative Colitis with Tacrolimus

clinical symptoms, colonoscopic findings, and results of biopsy, the case was diagnosed as severe pancolitis-type UC, and the patient was admitted for treatment.

He had no past medical history or allergy and no particular family medical history. His status on admission was: height, $183 \mathrm{~cm}$; body weight, $69 \mathrm{~kg}$; blood pressure, 116/67; pulse, $108 / \mathrm{min}$; body temperature, $36.8^{\circ} \mathrm{C}$. His defecation frequency was $8-10 /$ day with $4-5 /$ day melena and night defecation. No ulcers in the oral cavity, no congestion or swelling of the skin, and no ulceration of the vulva were found. The abdomen was flat and soft, and moderate spontaneous pain and tenderness were noted in the infraumbilical region.

Blood tests on admission showed anemia with $\mathrm{Hb}$ of $13.1 \mathrm{~g} / \mathrm{dL}$, leukocyte count $9,880 / \mu \mathrm{L}$, and high erythrocyte count of $58.3 \times 10^{4} / \mu \mathrm{L}$. Biochemical tests showed total protein of $6.4 \mathrm{mg} / \mathrm{dL}$; albumin $2.7 \mathrm{mg} / \mathrm{dL}$; creatinine $0.61 \mathrm{mg} / \mathrm{dL}$; low chloride level of 97 $\mathrm{mEq} / \mathrm{L}$, and high C-reactive protein level of $6.66 \mathrm{mg} / \mathrm{dL}$, indicating inflammatory reaction. Coagulation test showed decreased prothrombin time of 62\%, and high D-dimer of 2.0 $\mu \mathrm{g} / \mathrm{mL}$. Fecal culture showed indigenous bacteria only (Table 1 ).

He underwent colonoscopy, where the scope was advanced up to the transverse colon. Extensive undermining ulcers, severe mucosal edema, and scope contact-induced hemorrhage were observed in the transverse colon and over the sigmoid colon. Mucosal edema was noted in the rectum, but visible vascular patterns and erosion were only partially observed (Fig. 1).

\section{Course after Admission}

Treatment with 4,000 mg aminosalicylic acid (5-ASA) and prednisolone (PSL) at 60 $\mathrm{mg} /$ day was initiated after the diagnosis of UC. Following decreases in the defecation and melena frequencies to 6-7/day and 3-4/day, respectively, and alleviation of abdominal pain at day 3 after initiation of steroid treatment, the PSL dose was gradually decreased to 50 $\mathrm{mg} /$ day at day $6,40 \mathrm{mg} /$ day at day 11 , and $30 \mathrm{mg} /$ day at day 16 . However, after the last reduction, defecation and melena frequencies increased to 8-10/day and 4-5/day, respectively, and abdominal pain aggravated. Accordingly, the condition was considered steroiddependent intractable UC, and tacrolimus was administered. A high tacrolimus trough level $(10-15 \mathrm{ng} / \mathrm{dL})$ was achieved at day 2 of treatment, which was maintained during the course of treatment. Clinically, 2 weeks of treatment with tacrolimus reduced the defecation frequency to 5-6/day and melena frequency to 1-2/day, though night defecation was still reported, together with mild abdominal pain, but the general clinical condition showed some improvement.

Colonoscopy was performed to determine the effect of tacrolimus treatment at the end of week 2 . The scope was inserted and advanced to the end of the ileum. No mucosal congestion or erosion was noted at the end of the ileum. Bauhin's valve was slightly dilated. Mild mucosal edema was observed from the ileocecum to the ascending colon. Edema was moderate in the transverse colon to the sigmoid colon, and extensive ulcers were still observed, though the mucosa of the ulcer floor showed signs of regeneration and flattening compared with that on previous colonoscopy. In the rectum, only partial congestion was noted (Fig. 2).

At the end of week 4 of tacrolimus treatment, the clinical symptoms included further improvement in the frequency of defecation (to 2-3/day) and melena (to 0-1/day), no night defecation, and mild abdominal pain. Since the clinical condition improved, the tacrolimus dose was reduced to a low trough level (5-10 ng/dL). Another colonoscopy performed at the 


\section{Case Reports in Gastroenterology}

Case Rep Gastroenterol 2017;11:168-177

(c) 2017 The Author(s). Published by S. Karger AG, Basel www.karger.com/crg

to at: Development and Improvement of Simple Colonic Mucosal Ulcer during

Treatment of Severe Ulcerative Colitis with Tacrolimus

end of the 4-week tacrolimus treatment showed slight congestion of Bauhin's valve, mild mucosal edema from the ileocecum to the ascending colon, mild edema in the transverse colon to the sigmoid colon, with further regeneration and flattening of ulcer floor mucosa compared with that on the previous colonoscopy, persistent disappearance of rectal erosion as observed on previous colonoscopy, together with congested inflammatory polyps (Fig. 3).

Food ingestion was initiated. The clinical symptoms before initiation of food ingestion were frequency of defecation of 2-3/day and melena of $0-1$ /day, no night defecation, and no abdominal pain. Colonoscopy performed at the end of week 6 of tacrolimus treatment showed no congestion or erosion at the end of the ileum, slight dilation of Bauhin's valve, a new round ulcer in the inferior lip of Bauhin's valve, fewer areas with visible vascular patterns in the ileocecum to the ascending colon, ulcer scars and inflammatory polyps in the transverse colon to the sigmoid colon, partial improvement of the visible vascular patterns, and lack of erosion in the rectum (Fig. 4).

At that stage, Behçet's signs were absent, and no histopathological findings suggestive of BD were identified on the biopsy; accordingly, the new round ulcer in the inferior lip of Bauhin's valve was diagnosed as simple ulcer. The mucosal lesions found in the large intestine, other than those in the ileocecum, showed marked improvement. Accordingly, the PSL dose was gradually reduced, and the patient was discharged. The PSL dose at the time of discharge was $15 \mathrm{mg}$ and it was subsequently reduced to $10 \mathrm{mg}$. The clinical symptoms at discharge of defecation frequency of 2-3/day without melena or abdominal pain were considered to reflect clinical remission. Cytomegalovirus was negative throughout the hospital stay.

After discharge, the PSL dose was gradually reduced to discontinuation at the outpatient clinic, whereas tacrolimus and 5-ASA administrations were continued. Oral treatment with azathioprine was initiated at $25 \mathrm{mg} /$ day, but mild nausea developed making continuation of treatment difficult. Remission was clinically maintained. Colonoscopy performed at 12 weeks after the start of tacrolimus treatment showed erosion at the end of the ileum, slight dilation of Bauhin's valve, extension of the round ulcer compared with previous colonoscopy, partial congestion of the ileocecum to the ascending colon, improvement of visible vascular patterns, shrinking ulcer scars and inflammatory polyps in the transverse colon to the sigmoid colon, and complete healing of the rectal mucosa (Fig. 5).

No clinical relapse occurred thereafter and remission was maintained. Colonoscopy at the end of 18 weeks of tacrolimus treatment showed only small erosion and slight congestion in the ileocecum, and few areas of mucosal congestion and small erosions scattered in other regions of the large intestine (Fig. 5). A repeat colonoscopy at 36 weeks of tacrolimus treatment showed only slight congestion in the ileocecum and healing of the mucosa in other regions (Fig. 6). Throughout the clinical course, there were no signs of BD, and all tests and cultures for cytomegalovirus and stool specimens were negative. There was also no history of use of any nonsteroidal anti-inflammatory drugs.

\section{Discussion}

BD manifests itself in various systemic signs such as oral aphthus lesions, erythema nodosum, and iridocyclitis, as well as intestinal lesions [3], with the observation of ulcera- 
tion in the ileocecum in typical cases. Simple ulcers in the ileocecum similar to those found in $\mathrm{BD}$ are also observed in other conditions, but no other findings are observed in other organs, i.e., simple ulcers are ulcer lesions in the ileocecum not meeting the diagnostic criteria of BD. The histopathological features were also similar and differentiation of these diseases is difficult. Ulcerative lesions may develop in the entire digestive tract in BD. Serial examination is important because development of lesions similar to UC as pre-lesions in the large intestine of patients with BD has been reported [4].

The cause and mechanism of development of simple lesions during treatment of UC are still unclear. However, several studies reported that infliximab was effective for intestinal BD and simple ulcers in Japan, and the possibility of involvement of cytokines in the development of simple ulcers has been investigated [4-10], but to our knowledge, no study has reported the effectiveness of tacrolimus for simple ulcers.

The clinical features, such as bleeding and abdominal pain, did not worsen in our patients following the development of simple ulcer; rather, clinical improvement was evident. It is possible the tacrolimus treatment induced the development of simple ulcer based on the course of this case. However, healing of the simple ulcer following continuation of tacrolimus treatment was confirmed. The present case suggests that tacrolimus therapy can result in healing and resolution of simple ulcers. Further close follow-up, including clinical and colonoscopic examinations, is necessary in this case [11].

\section{Conclusion}

We described a patient who developed a simple round ulcer in the inferior lip of Bauhin's valve during tacrolimus treatment of severe UC. The simple ulcer improved with continuation of tacrolimus treatment.

\section{Statement of Ethics}

The work done for this paper has the approval of the Institutional Review Board and all rules were followed.

\section{Disclosure Statement}

The authors have no conflict of interest.

\section{References}

1 The Research Group of Intractable Inflammatory Bowel Disease Affiliated with the Japan Ministry: Research on chronic nonspecific multiple ulcers of the small intestine. The Assignation Research Reports in Fiscal 2015 by the Group of Suzuki.

2 The Research Group of Intractable Inflammatory Bowel Disease Affiliated with the Japan Ministry: Research on ulcerative colitis. The Assignation Research Reports in Fiscal 2015 by the Group of Suzuki. 


\section{Case Reports in Gastroenterology}

\begin{tabular}{l|l}
\hline Case Rep Gastroenterol 2017;11:168-177 \\
\hline DOI: $10.1159 / 000456605$ & $\begin{array}{l}\text { (c) 2017 The Author(s). Published by S. Karger AG, Basel } \\
\text { www.karger.com/crg }\end{array}$ \\
\hline
\end{tabular}

Ito et al.: Development and Improvement of Simple Colonic Mucosal Ulcer during Treatment of Severe Ulcerative Colitis with Tacrolimus

3 The Research Group of Intractable Inflammatory Bowel Disease Affiliated with the Japan Ministry: Research on Behcet's disease. The Assignation Research Reports in Fiscal 2015 by the Group of Suzuki.

-4 Yamagata K, Ishiguro Y, Tanaka M, et al.: Characteristic future of intestinal Behcet's disease and simple ulcer. Jap J Clin Med 2012;70:573-580.

5 Iwao Y, Inoue N, Sujino T, et al: Courses of intestinal Behcet's disease and simple ulcer with treatment: cases treated with anti-TNF- $\alpha$ antibody (infliximab). Stomach and Intestine 2011;46:1051-1059. Hamzaoui K, et al: Production of TNF-alpha and IL-1 in active Behcet's disease. J Rheumatol 1990;17:1428-1429.

7 Naganuma M, et al: Efficacy of infliximab for induction and maintenance of remission in intestinal Behçet's disease. Inflamm Bowel Dis 2008;14:1259-1264.

$\checkmark 8$ Kishi M, et al: A case of a simple ulcer associated with ulcerative colitis that demonstrated a good clinical response to infliximab. Jap Soc Gastroenterol 2015;112:1682-1688.

9 Ono M, Murakami K, Oda M, et al: A case of simple ulcer with ulcerative colitis. Jap J Gastroenterol 2005;102:894-899.

10 Naoki Y, et al: The clinical efficacy of thalidomide and infliximab therapy in steroid refractory intestinal Behcet's disease, report of two cases. Stomach and Intestine 2011;46:1067-1072.

11 Murano S, Murano N, Abe Y, et al: Long-term clinical outcome and treatment response of intestinal Behcet's disease and simple ulcer. Stomach and Intestine 1979;14:749-767.
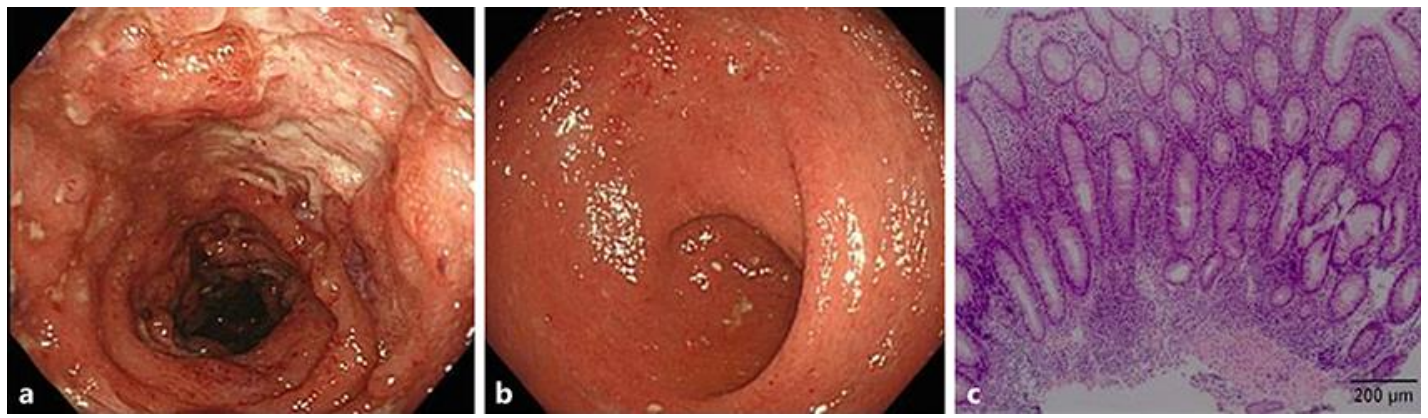

Fig. 1. Colonoscopy findings before treatment: observation up to the transverse colon. Extensive undermining ulcers, severe mucosal edema, and scope contact-induced hemorrhage were observed in the transverse colon (a) over the sigmoid colon. Although mucosal edema was present in the rectum (b), only partially visible vascular patterns and erosion were observed. Results of rectal biopsy (c): mucosa with relatively marked inflammatory cell infiltration including neutrophils and regenerative changes. No epithelioid granuloma or active vasculitis was observed. The histopathological diagnosis was ulcerative colitis with mild activity, compatible ulcerative colitis. Diffuse inflammatory cell infiltration $(++)$, reduction of germinal (goblet suspicion) cells $(+)$, regressive change of glandular epithelium $( \pm)$, crypt abscess $(-)$, disturbed gland duct arrangement $(+)$, gland regeneration $(+)$, Paneth cells $(-)$. 


\section{Case Reports in Gastroenterology}
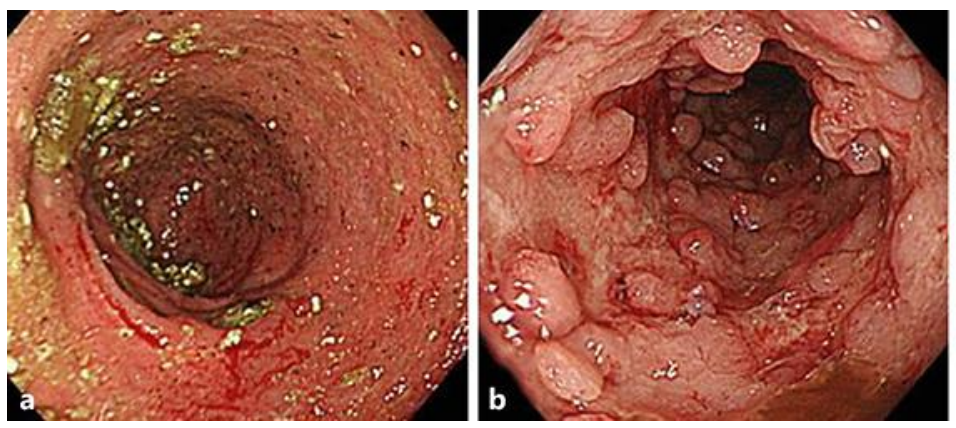

Fig. 2. Colonoscopy findings at week 2 of tacrolimus administration. The scope was inserted up to the end of the ileum. No reddening or erosion was noted at the end of ileum. Bauhin's valve was slightly dilated. In the ileocecum (a) over the ascending colon, mild mucosal edema was observed. In the transverse colon (b) over the sigmoid colon, edema was moderate and extensive ulcers were observed, but the mucosa of the ulcer floor was regenerated and flattened. Only partial reddening was noted (c).
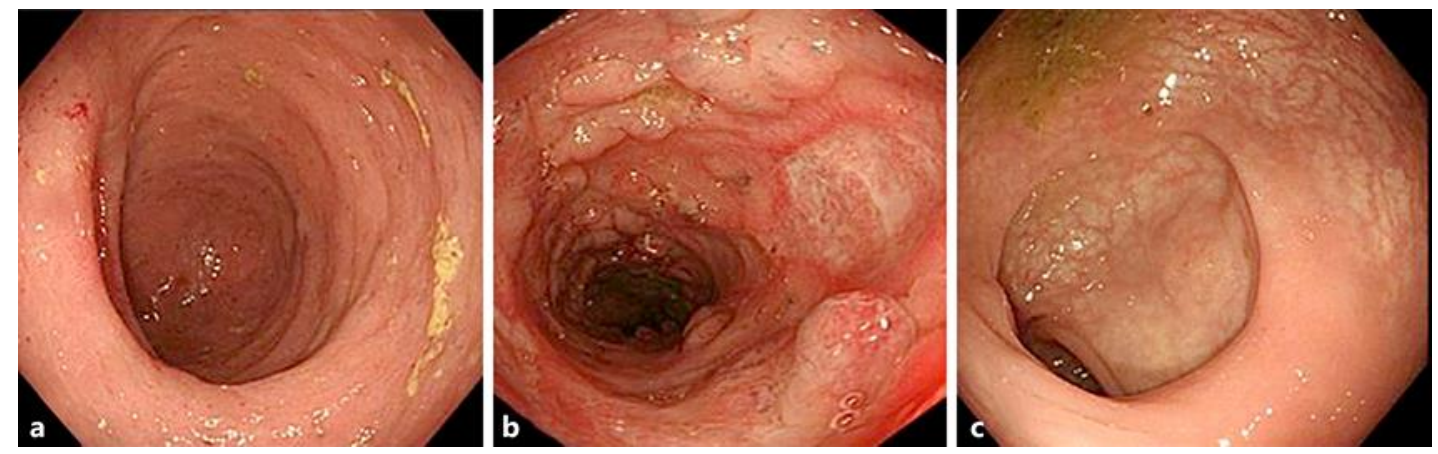

Fig. 3. Colonoscopy findings at week 4 of tacrolimus administration. The scope was inserted up to the cecum. Bauhin's valve was slightly dilated. In the ileocecum (a) over the ascending colon, mild mucosal edema was observed. In the transverse colon (b) over the sigmoid colon, edema was moderate, the mucosa of the floor of the extensive ulcers was further regenerated and flattened, and reddening inflammatory polyps were noted. Rectal erosion had resolved as observed on the previous colonoscopies (c). 


\section{Case Reports in Gastroenterology}
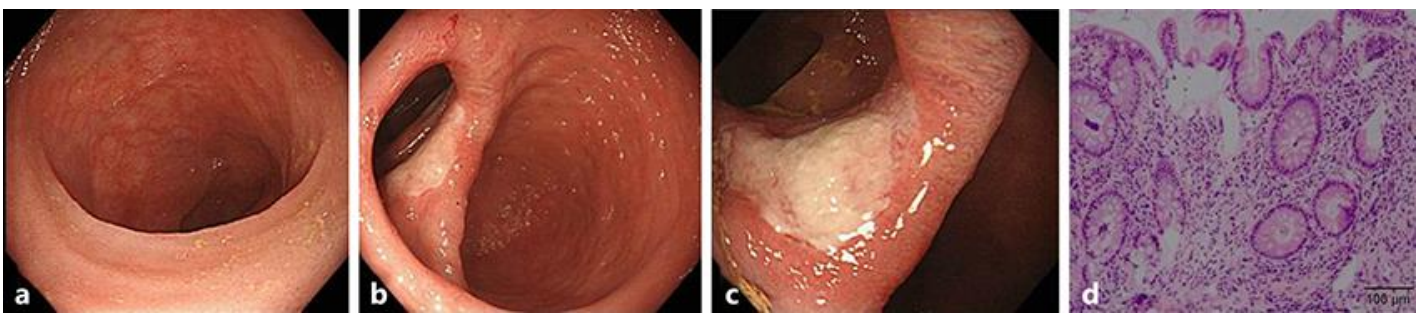

Fig. 4. Colonoscopy findings at week 6 of tacrolimus administration. The scope was inserted up to the end of the ileum. No reddening or erosion was observed at the end of the ileum (a). Bauhin's valve (b) was slightly dilated, and a round ulcer was formed in the inferior lip of Bauhin's valve. In the ileocecum over the ascending colon, visible vascular patterns were reduced. In the transverse colon (c) over the sigmoid colon, ulcer scars and inflammatory polyps were noted. Visible vascular patterns were partially improved. Rectal erosion had resolved as observed on the previous colonoscopies. Results of biopsy from the margin of the ulcer in Bauhin's valve (d): the mucosa showed mild activity. Gland tissue showed extension and slight dentate changes, but the nuclei were small. Diffuse inflammatory cell infiltration $(+)$, decrease in germinal (goblet suspicion) cells $( \pm)$, regressive change of glandular epithelium $(-)$, crypt abscess $(-)$, disturbed gland duct arrangement $(+)$, gland regeneration $(+)$, Paneth cells $(-)$.
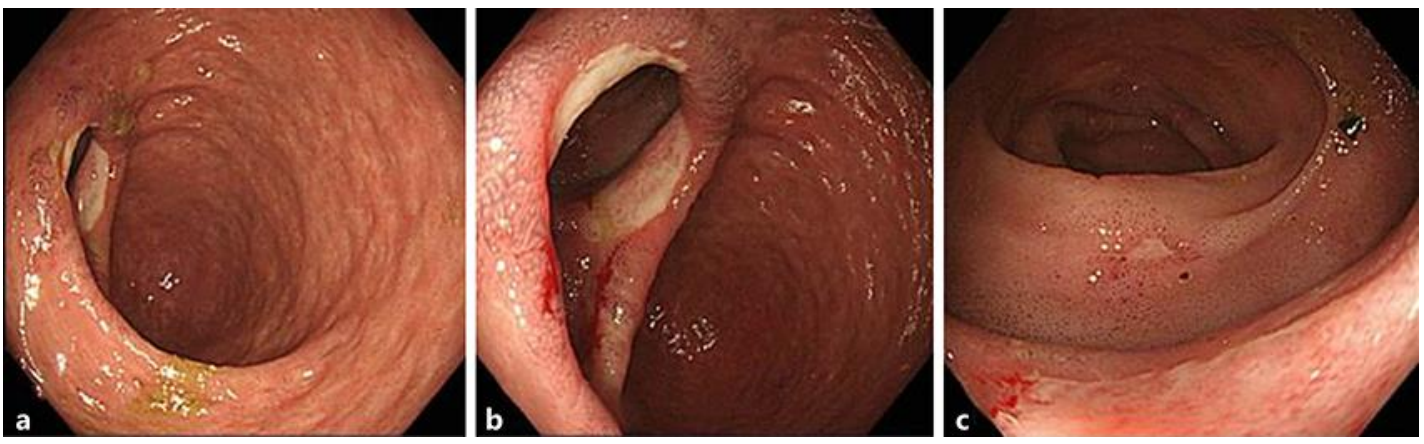

Fig. 5. Colonoscopy findings at week 12 of tacrolimus administration. The scope was inserted up to the end of the ileum. Erosion was present at the end of the ileum (c). Bauhin's valve (a, b) was slightly dilated, and the round ulcer expanded compared with that on the previous colonoscopies. In the ileocecum over the ascending colon, reddening was partially noted, but visible vascular patterns were improved. In the transverse colon (b) over the sigmoid colon, ulcer scars and inflammatory polyps were noted but shrunk compared with those on the previous colonoscopies. Healed mucosa was noted in the rectum. 


\section{Case Reports in Gastroenterology}
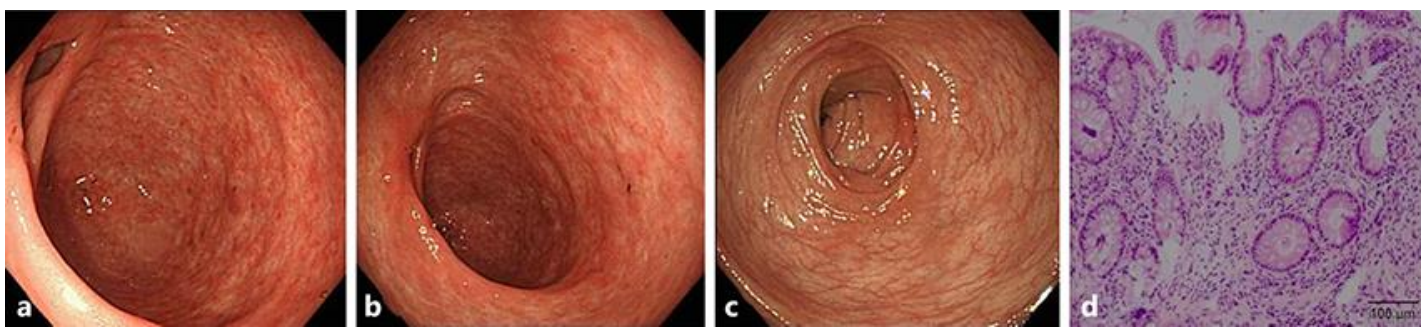

Fig. 6. Colonoscopy findings at week 18 of tacrolimus administration. The scope was inserted up to the end of the ileum. Reddening and erosion were absent at the end of the ileum (c). Bauhin's valve (a, b) was slightly dilated, and small erosion was noted. Biopsy 1: mucosal atrophy was noted in the ileocecum over the ascending colon. In the transverse colon (b) over the sigmoid colon, ulcer scars, inflammatory polyps, and partial mucosal healing were observed. Healed mucosa was noted in the rectum. Results of biopsy of erosion collected from Bauhin's valve (d): mucosa with very mild activity. Diffuse inflammatory cell infiltration $(+)$, decrease in germinal (goblet suspicion) cells $( \pm)$, regressive change of glandular epithelium $(-)$, crypt abscess (-), disturbed gland duct arrangement $(+)$, gland regeneration $(+)$, Paneth cells $(-)$. 


\section{Case Reports in Gastroenterology}

\begin{tabular}{l|l}
\hline Case Rep Gastroenterol 2017;11:168-177 \\
\hline DOI: 10.1159/000456605 & $\begin{array}{l}\text { (c) 2017 The Author(s). Published by S. Karger AG, Basel } \\
\text { www.karger.com/crg }\end{array}$ \\
\hline
\end{tabular}

Ito et al.: Development and Improvement of Simple Colonic Mucosal Ulcer during

Treatment of Severe Ulcerative Colitis with Tacrolimus

Table 1. Blood test findings on admission

\begin{tabular}{ll}
\hline WBC & $9.88 \times 10^{3} / \mu \mathrm{L}$ \\
\multicolumn{1}{c}{ NEUT } & $68.3 \%$ \\
\multicolumn{1}{l}{ LYMPH } & $112.8 \%$ \\
$\mathrm{Hb}$ & $13.1 \mathrm{~g} / \mathrm{dL}$ \\
Plt & $58.3 \times 10^{4} / \mu \mathrm{L}$ \\
\hline Blood coagulation & \\
PT\% & \\
Fib & $62.9 \%$ \\
D-dimer & $530 \%$ \\
\hline Special blood examination & $2.0 \mu \mathrm{g} / \mathrm{mL}$ \\
TP & \\
ALB & $6.4 \mathrm{~g} / \mathrm{dL}$ \\
T-Bil & $2.9 \mathrm{~g} / \mathrm{dL}$ \\
AST & $0.6 \mathrm{mg} / \mathrm{dL}$ \\
ALT & $16 \mathrm{U} / \mathrm{L}$ \\
ALP & $30 \mathrm{U} / \mathrm{L}$ \\
$\gamma$ GTP & $260 \mathrm{U} / \mathrm{L}$ \\
BUN & $82 \mathrm{U} / \mathrm{L}$ \\
Cre & $7.9 \mathrm{mg} / \mathrm{dL}$ \\
eGFR & $0.55 \mathrm{mg} / \mathrm{dL}$ \\
Na & $135.5 \mathrm{~mL} / \mathrm{min}$ \\
K & $135 \mathrm{mEq} / \mathrm{L}$ \\
CL & $3.7 \mathrm{mEq} / \mathrm{L}$ \\
TG & $97 \mathrm{U} / \mathrm{L}$ \\
HDL & $122 \mathrm{mg} / \mathrm{dL}$ \\
CRP & $26 \mathrm{mg} / \mathrm{dL}$ \\
Infection & $6.66 \mathrm{mg} / \mathrm{dL}$ \\
CMVAgC7-HRP & \\
T-SPOT.Tb & $(-)$ \\
Fecal culture & $(-)$ \\
\hline
\end{tabular}

ALB, albumin; ALP, alkaline phosphatase; ALT, alanine transaminase; AST, aspartate aminotransferase; BUN, blood urea nitrogen; CL, chlorine; CMVAgC7-HRP, C7 antigenemia; Cre, creatinine; CRP, C-reactive protein; eGFR, estimated glomerular filtration rate; Fib, fibrinogen; $\mathrm{Hb}$, hemoglobin; HDL, high-density lipoprotein cholesterol; K, potassium; LYMPH, lymphocytes; Na, sodium; NEUT, neutrophils; Plt, platelets; PT, prothrombin time; T-Bil, total bilirubin; TG, triglyceride; TP, total protein; WBC, white blood cell; $\gamma \mathrm{GTP}, \gamma$-glutamyltransferase. 